\title{
Efficacy of medical therapy in the initial management of acute primary angle closure in Asians
}

N Ramli',2, SM Chai', GS Tan', R Husain', S-T Hoh', C-L Ho ${ }^{1}$ and T Aung ${ }^{1,3}$

\begin{abstract}
Purpose Recent studies have advocated the use of laser iridoplasty or paracentesis in the initial management of patients with acute primary angle closure (APAC). The aim of this study was to ascertain the effectiveness of medical treatment consisting of topical and systemic intraocular pressure (IOP)-lowering agents in the initial management of APAC.
\end{abstract}

Methods This was an observational case series of consecutive patients presenting with APAC at a Singapore hospital over 2 years. On diagnosis, all subjects received intravenous acetazolamide followed by oral acetazolamide, topical pilocarpine, timolol, and steroid eye drops. Resolution of APAC was defined as IOP $<21 \mathrm{~mm} \mathrm{Hg}$ with no acute symptoms. Results In all, 134 consecutive APAC subjects were studied. The majority of subjects were Chinese (96.3\%) and female $(80 \%)$, and the mean age was $63.7 \pm 9.6$ years.

The mean presenting IOP was $58 \pm 12.7 \mathrm{~mm} \mathrm{Hg}$ and mean duration of symptoms was $2.8 \pm 3.2$ days. With medical therapy, APAC attacks resolved within $3,6,12$, and $24 \mathrm{~h}$ in $28(21.5 \%)$, $58(44.6 \%), 99(76.2 \%)$, and 116 (89.2\%) subjects, respectively. After resolution of APAC, laser iridotomy was performed in $81.6 \%$ of the subjects; $16.2 \%$ of the subjects underwent cataract extraction. There was failure of resolution of APAC in only 3 subjects $(2.2 \%)$. No subject suffered any serious side effects as a result of treatment. Conclusions Medical therapy resulted in resolution of APAC within $12 \mathrm{~h}$ in $76.2 \%$ of the subjects and within $24 \mathrm{~h}$ in $89.2 \%$ of the subjects, showing the effectiveness of medical therapy in the initial management of APAC.

Eye (2010) 24, 1599-1602; doi:10.1038/eye.2010.92; published online 25 June 2010
Keywords: angle closure glaucoma; open-angle glaucoma; intraocular pressure; acute primary angle closure

Introduction

Acute primary angle closure (APAC) is a potentially blinding ocular condition, occurring with high incidence in East Asians. ${ }^{1}$ Conventional treatment involves the administration of topical and systemic medications to reduce the intraocular pressure (IOP) in the initial stage, with a laser peripheral iridotomy performed to relieve pupillary block when corneal clarity permits. ${ }^{2}$

Recent studies have advocated the use of laser iridoplasty, which mechanically pulls open the angle by causing contraction of the peripheral iris to allow increased aqueous outflow, or anterior chamber paracentesis to remove aqueous, in the initial management of patients with APAC. ${ }^{3-5}$ The rationale for these methods is to lower the IOP rapidly to limit damage to the optic nerve and anterior segment structures. However, these methods may be associated with serious risks such as corneal decompensation, iris and lens damage, infection, or suprachoroidal haemorrhage.

Medical therapy of APAC is itself associated with some systemic and ocular risks. For example, carbonic anhydrase inhibitors may cause metabolic acidosis and electrolyte imbalances. Mannitol may induce congestive heart failure in susceptible individuals. The aim of this study was to ascertain the effectiveness and safety of medical treatment consisting of topical and systemic IOP-lowering agents in the initial management of APAC in an Asian population.

\section{Materials and methods}

This was an observational case series of consecutive patients presenting with APAC
${ }^{1}$ Singapore National Eye Centre and Singapore Eye Research Institute, Singapore

${ }^{2}$ University of Malaya, Kuala Lumpur, Malaysia

${ }^{3}$ Yong Loo Lin School of Medicine, National University of Singapore, Singapore

Correspondence: T Aung, Singapore National Eye Centre and Singapore Eye Research Institute, 11 Third Hospital Avenue, Singapore 168751, Singapore Tel: + 6591088561 . Fax: + 6562263395. E-mail: tin11@pacific.net.sg

Received: 4 February 2010 Accepted in revised form: 10 May 2010 Published online: 25 June 2010 
at a Singapore hospital over 2 years. Written informed consent was obtained from all subjects and the study had the approval of the ethics committee of the Singapore National Eye Centre, and was performed according to the tenets of the Declaration of Helsinki.

The following criteria were used to define cases of APAC:

1. Presence of at least two of the following symptoms: ocular or periocular pain, nausea and/or vomiting, and an antecedent history of intermittent blurring of vision and/or haloes.

2. Presenting IOP of $>28 \mathrm{~mm} \mathrm{Hg}$ (as measured by Goldmann applanation tonometry) in phakic eyes with the presence of at least three of the following signs: conjunctival injection, corneal epithelial oedema, mid-dilated unreactive pupil, and shallow anterior chamber (defined as Van Herick grade II or less).

On diagnosis, subjects without a history of sulphonamide allergy underwent a standardized protocol of management in which they received intravenous acetazolamide $(500 \mathrm{mg}$ ), followed by oral acetazolamide (250 mg) four times daily, topical pilocarpine (4\%) four times a day, and timolol (0.5\%) twice daily as well as betamethasone or prednisolone acetate every $3 \mathrm{~h}$ applied to the affected eye. If the IOP did not reduce after $2 \mathrm{~h}$ of this standard treatment, other medications such as intravenous mannitol, oral glycerol, topical latanoprost, or brimonidine were added. Patients were reviewed at regular intervals for IOP control, and resolution of APAC was defined as IOP $<21 \mathrm{~mm} \mathrm{Hg}$ with resolution of acute symptoms. Subjects were also observed for adverse effects of the medical therapy.

\section{Statistical analysis}

Predictive factors that may affect the resolution of APAC within 6, 12, and $24 \mathrm{~h}$ were analysed. These were gender, age, presenting IOP, and symptom duration before the presentation. A univariate and multivariate analysis of the association of each factor with the resolution of APAC episode was carried out. Odds ratio was calculated along with their $95 \%$ confidence intervals (CIs).

\section{Results}

A total of 134 consecutive APAC subjects (136 eyes) were studied. The demographic features of these subjects are summarized in Table 1. The majority of subjects were Chinese $(96.3 \%)$ and female $(80 \%)$, and the mean age was $63.7 \pm 9.6$ years. The mean presenting IOP was $58 \pm 12.7 \mathrm{~mm} \mathrm{Hg}$ and mean duration of symptoms was $2.8 \pm 3.2$ days. With medical therapy, APAC episodes resolved within $3,6,12$, and $24 \mathrm{~h}$ in $28(21.5 \%)$,
Table 1 Demographic and clinical characteristics of subject with acute primary angle closure in this study

\begin{tabular}{lc}
\hline $\begin{array}{l}\text { Age (years) } \\
\text { Mean }\end{array}$ & 63.9 \\
Range & $42-83$ \\
Sex & \\
$\quad$ Male & $27(20 \%)$ \\
Female & $107(80 \%)$ \\
Race & \\
Chinese & $129(96.3 \%)$ \\
Malay & $4(3 \%)$ \\
Indian & $1(0.7 \%)$ \\
Mean presenting IOP (mm Hg) & $58 \pm 12.7$ \\
Duration of symptoms (days) & Mean $2.8(\mathrm{SD} 3.2)$ \\
& (range $1 \mathrm{~h}-21$ days) \\
& Median 2.0 \\
\hline
\end{tabular}

$58(44.6 \%), 99(76.2 \%)$, and $116(89.2 \%)$ subjects, respectively. In all, 55 patients $(41 \%)$ required intravenous mannitol and additional IOP-lowering medications as described above. No serious side effects of medical treatment were observed in this series.

After resolution of APAC, visual acuity was $6 / 12$ or better in 68 subjects $(50.7 \%), 6 / 15$ to $6 / 48$ in 42 subjects $(31.3 \%)$, and $6 / 60$ or worse in 24 subjects $(17.9 \%){ }^{6}$ LPI was performed in $81.6 \%$, and $16.2 \%$ of the subjects underwent cataract extraction within 1 week to 2 months after the initial APAC episode without LPI.

There was failure of resolution of APAC in only three subjects $(2.2 \%)$. Of these, two subjects had symptoms for $48 \mathrm{~h}$ while the third subject had symptoms for only $3 \mathrm{~h}$ before presentation. The presenting IOP in these patients was between $43-68 \mathrm{~mm} \mathrm{Hg}$. All three subjects could not undergo laser peripheral iridotomy because of poor cornea clarity and they required glaucoma surgery within $72 \mathrm{~h}$ of admission. The fellow eyes in all the three cases were pseudophakic.

Univariate and multivariate analyses failed to show any statistically significant patient or clinical factors (gender, age, presenting IOP, or duration of symptoms) that were associated with resolution of the APAC episodes within or beyond $12 \mathrm{~h}$ (Table 2 ). Similar results were obtained for APAC episodes resolving within or beyond $6 \mathrm{~h}$, or those that resolved within or beyond $24 \mathrm{~h}$ (data not shown).

\section{Discussion}

Our results show that medical treatment of APAC is effective in lowering IOP in a safe and efficient manner. The majority of our patients $(89.2 \%)$ had their APAC episode aborted within $24 \mathrm{~h}$ just on medication alone. APAC occurs secondary to pupil block; hence medical treatment is only 
Table 2 Factors affecting time taken for resolution of acute primary closure episode (within or beyond $12 \mathrm{~h}$ )

\begin{tabular}{|c|c|c|c|c|c|c|}
\hline \multirow[t]{2}{*}{ Risk factors } & \multicolumn{2}{|c|}{ Time taken to resolve } & \multirow{2}{*}{$\begin{array}{c}\text { Odds ratio } \\
\text { (univariate analysis) }\end{array}$} & \multirow[t]{2}{*}{$95 \% C I$} & \multirow{2}{*}{$\begin{array}{c}\text { Odds ratio } \\
\text { (multivariate analysis) }\end{array}$} & \multirow[t]{2}{*}{$95 \% C I$} \\
\hline & $<12 h$ & $>12 h$ & & & & \\
\hline \multicolumn{7}{|l|}{ Gender } \\
\hline Male & 22 & 6 & 1.00 & & 1.00 & \\
\hline Female & 77 & 26 & 1.24 & $0.45-3.39$ & 0.76 & $0.24-2.4$ \\
\hline \multicolumn{7}{|l|}{ Age } \\
\hline$\leqslant 60$ years & 33 & 11 & 1.00 & & 1.00 & \\
\hline$>60$ years & 66 & 21 & 0.96 & $0.41-2.21$ & 1.15 & $0.48-2.76$ \\
\hline \multicolumn{7}{|c|}{ Presenting IOP $(\mathrm{mm} \mathrm{Hg})$} \\
\hline $21-60$ & 49 & 15 & 1.00 & & 1.00 & \\
\hline$>60$ & 50 & 16 & 1.05 & $0.47-2.34$ & 1.09 & $0.48-2.51$ \\
\hline \multicolumn{7}{|c|}{ Symptom duration } \\
\hline$<3$ days & 72 & 24 & 1.00 & & 1.00 & \\
\hline$>3$ days & 23 & 6 & 0.75 & $0.28-2.06$ & 1.22 & $0.42-3.5$ \\
\hline
\end{tabular}

Abbreviations: CI, confidence interval; IOP, intraocular pressure.

a temporizing measure until definitive treatment by laser peripheral iridotomy is performed. Surprisingly, factors such as presenting IOP and duration of symptoms before presentation did not seem to affect the time taken for resolution of the attack from medical therapy.

Studies in recent years have advocated the use of laser iridoplasty or anterior chamber paracentesis in the initial management of APAC. ${ }^{3-5}$ In a randomized trial comparing argon laser peripheral iridoplasty with conventional systemic IOP-lowering medications as the first-line treatment of APAC, the laser peripheral iridoplasty group had a lower IOP as compared with the medical treatment group. This difference was significant only within the first $2 \mathrm{~h}$ after treatment, and thereafter the difference was not statistically significant. ${ }^{3}$ Of note, laser peripheral iridoplasty could be associated with corneal burns, damage to corneal endothelial cells, and iris atrophy. ${ }^{6,7}$ Anterior chamber paracentesis is an invasive intraocular procedure that could present technical difficulties if the anterior chamber is very shallow, thus increasing the risk of iris or lens damage. The patient may not be able to cooperate because of the presenting symptoms of headache, nausea, or pain, thus increasing the risk of complications from this procedure. ${ }^{5}$ A certain level of experience in the attending physician performing the procedure is required. In a case series by Lam et al, ${ }^{4}$ patients with APAC who were treated with immediate paracentesis and medications had a significantly lower IOP in the first hour after treatment, as compared with those who were treated with conventional therapy. However, by $2 \mathrm{~h}$ it was observed that the paracentesis group had a mean IOP that approached that of the conventional therapy group.
There was no significant adverse event in our group of patients treated medically. Although it has been argued in the recent literature that the advantages of interventions such as ALPI and paracentesis are that it lowers the IOP faster (and hence causes less permanent damage to the optic nerve and other ocular tissues), there is currently no evidence that shortening the duration of the acute attack results in less optic nerve damage in the long term. Iester $e t a l^{8}$ investigated RNFL thickness measured with the confocal scanning laser polarimeter in patients undergoing LASIK who had induced IOP of up to $100 \mathrm{~mm} \mathrm{Hg}$ for $45 \mathrm{~s}$ and found no decrease in RNFL thickness up to a month later. Furthermore, a study on the long-term outcomes of APAC patients in Singapore found that the delay in presentation of more than 3 days was not a significant risk factor in later development of chronic IOP rise after the initial APAC episode. ${ }^{9}$ Although it seems biologically plausible that rapid lowering of IOP after APAC should cause less optic nerve damage compared with a more gradual reduction, this has yet to be proven in clinical studies. Furthermore, the risk to ocular morbidity of such strategies is likely to be greater. Considering the fact that most APAC attacks are initially seen by residents and more junior staff on call, medical therapy alone should remain as the initial first-line treatment. In fact, at relatively low IOP levels such as below $30 \mathrm{~mm} \mathrm{Hg}$, pilocarpine may be all that is required to abort the APAC episode.

Our study is limited by the fact that it was a noncomparative study. The population studied was mainly of Chinese descent; hence, the figures may only be reflective of patients in this population. It has been 
postulated that angle closure glaucoma may have differences in aetiology and mechanisms between East Asian and Caucasian people. ${ }^{10}$ Another limitation is that although the medical management of APAC is described in textbooks, the actual protocol may differ in different eye institutions. There is a lack of evidence detailing how much each component contributes towards IOP lowering in the acute stage. Choong et $a l^{11}$ used a similar protocol in his study but the patient had to lie supine for an hour and showed $100 \%$ response rate within $7 \mathrm{~h}$. We did not have optic disc or visual field information and it would be important to investigate whether those who took longer to resolve had worse outcomes in the long term. Another limitation was that we did not measure anterior chamber depth, which may have been a factor influencing response to medical treatment.

In conclusion, this study shows that medical therapy is safe, efficacious, and still has an important role in the initial management of patients presenting with APAC. Until other strategies are shown to be superior in terms of resulting in reduced optic nerve damage as well as safer in terms of acute and long-term complications, medical management of APAC should remain as first-line treatment.

\section{Summary}

What was known before

- Recent studies have advocated paracentesis and iridoplasty instead of medical therapy as first-line treatment for acute primary angle closure.

\section{What this study adds}

- Our study has found that medical therapy is safe and efficacious as first-line treatment for acute angle closure in an Asian population.

\section{Conflict of interest}

The authors declare no conflict of interest.

\section{Acknowledgements}

This work was supported by grants from the National Medical Research Council, Singapore and National Medical Research Foundation, Singapore.

\section{References}

1 Seah SK, Foster PJ, Chew PT, Jap A, Oen F, Fam HB et al. Incidence of acute primary angle-closure glaucoma in Singapore. An island-wide survey. Arch Ophthalmol 1997; 115: $1436-1440$.

2 Saw SM, Gazzard G, Friedman DS. Interventions for angleclosure glaucoma: an evidence-based update. Ophthalmology 2003; 110: 1869-1878.

3 Lam DS, Lai JS, Tham CC, Chua JK, Poon AS. Argon laser peripheral iridoplasty versus conventional systemic medical therapy in treatment of acute primary angle-closure glaucoma: a prospective, randomized, controlled trial. Ophthalmology 2002; 109: 1591-1596.

4 Lam DS, Chua JK, Tham CC, Lai JS. Efficacy and safety of immediate anterior chamber paracentesis in the treatment of acute primary angle-closure: a pilot study. Ophthalmology 2002; 109: 64-70.

5 Lam DS, Tham CC, Lai JS, Leung DY. Current approaches to the management of acute primary angle closure. Curr Opin Ophthal 2007; 18: 146-151.

6 Tan GS, Hoh ST, Husain R, Gazzard G, Oen FT, Seah SK et al. Visual acuity after acute primary angle closure and considerations for primary lens extraction. $\mathrm{Br} J$ Ophthalmol 2006; 90(1): 14-16.

7 Ritch R, Tham CC, Lam DS. Argon laser peripheral iridoplasty (ALPI): an update. Surv Ophthalmol 2007; 52: 279-288.

8 Iester M, Tizte P, Mermoud A. Retinal nerve fiber layer thickness changes after an acute increase in intraocular pressure. J Cataract Refract Surg 2002; 28: 2117-2122.

9 Aung T, Ang LP, Chan SP, Chew PTK. Acute primary angle closure: long term intraocular pressure outcome in Asian eyes. Am J Ophthalmol 2001; 131: 7-12.

10 He M, Foster PJ, Johnson GJ, Khaw PT. Angle-closure glaucoma in East Asian and European people. Different diseases? Eye 2006; 20: 3-12.

11 Choong YF, Irfan S, Menage MJ. Acute angle closure glaucoma: an evaluation of a protocol for acute treatment. Eye 1999; 13: 613-616. 